\title{
Image Segmentation between Crop and Weed using Hyperspectral Imaging for Weed Detection in Soybean Field
}

\author{
Yumiko Suzuki, Hiroshi Oкамото* and Takashi KataOKA* \\ Graduate School of Agriculture, Hokkaido University, Kita-9, Nishi-9, Kita-ku, Sapporo 060-8589, Japan \\ * Research Faculty of Agriculture, Hokkaido University, Kita-9, Nishi-9, Kita-ku, Sapporo 060-8589, Japan
}

(Received June 13, 2008)

\begin{abstract}
The goal of this study was image segmentation between crop and weed using hyperspectral imaging for weed detection. This technique is useful for selective weeding using spot spraying or a robotic system. A hyperspectral image consists of a large number of pixel spectra. Therefore, image segmentation was executed pixel by pixel. After each pixel spectrum was extracted from the image, it was identified as soil or plant. If the pixel spectrum was identified as plant, it was categorized as crop or weed. For the pixel discriminant model between soil and plant, simple NDVI thresholding was employed. The pixel discriminant model between crop and weed consisted of normalization, generation of explanatory variables and discrimination, and four types of models were developed and validated. Finally, segmented images between crop and weed were generated from the hyperspectral images by applying these models. As the validation results, pixel discrimination between soil and plant was performed with $99.9 \%$. Also, most pixel discriminant models between crop and weed had a success rate of more than $90 \%$. As a result of image segmentation between crop and weed, most hyperspectral images were segmented correctly. This study demonstrated the possibility of weed detection using hyperspectral imaging.
\end{abstract}

Keywords: image processing, linear discriminant analysis, machine vision, multiple-layered neural network, plant classification, principal component analysis

\section{INTRODUCTION}

A reduced and aging labor force is a serious problem in Japan's agricultural industries, and therefore efficient agricultural systems need to be developed. Weed control, which is very important in field management, is conducted by means of chemical, biological and physical methods. Although chemical methods using herbicides are highly efficient, numerous problems are posed such as residual agricultural chemicals in crops and soil, pollution of air and underground water and damage to farmers' health. Biological methods are safer because they do not use herbicides, but the work is intense due to the use of plants and other living things. On the other hand, physical methods are safe for the environment, crops and farmers. Weeds between crop rows (inter-row) can be efficiently removed by using an agricultural implement such as a cultivator. However, weeds around each crop (intra-row) are mainly removed manually. Consequently, there is an increasing demand for a robotic weeding system for efficient weed control such as by spot treatment around each crop.

In some studies, machine vision with RGB color imaging or multispectral imaging was em-

Corresponding author: Yumiko Suzuki, fax: +81-11-706-2555, e-mail : yumisuzuki@bpe.agr.hokudai.ac.jp 


\section{Y. SUZUKI ET AL.}

ployed for a robotic weeding system. Steward and Tian (1999) developed a system to detect crop inter-row edges and to estimate the number of weeds. Terawaki et al. (2002) discriminated between sugar beet and weed by using discriminant analysis and the angle of the leaf tip portion. Yang et al. (2002) developed a weed recognition system based on artificial neural networks in a corn field. Tang et al. (2003) discriminated between broadleaf and grass weed by using wavelets and neural networks. Bajwa and Tian (2001) generated a spatial distribution map of weed density by using multispectral imaging in a soybean field. Yang et al. (2004) discriminated between crop and weed by using multispectral imaging with decision tree analysis.

In a number of recent studies, hyperspectral imaging was also employed for weed detection. This imaging has the possibility of weed detection at a high rate of accuracy because the image contains more detailed spectral information compared to the RGB color image and multispectral image. At the airborne base, Goel et al. (2003) detected weed infestations in a corn field by using seven classification methods, and Karimi et al. (2005) obtained a model for detecting weeds in a corn field by using discriminant analysis, artificial neural networks and decision tree analysis.

Although airborne hyperspectral imaging could obtain weed density and existence on a large scale, detection and classification of individual weeds would be difficult due to its low spatial resolution. On the other hand, ground-based imaging would be able to detect and classify individual weeds due to the high spatial resolution. In studies using ground-based hyperspectral imaging, Iino et al. (2005) obtained the pixel discriminant models between sugar beet and weeds using Euclidean and Mahalanobis distance, and Okamoto et al. (2007) developed a pixel discriminant model for weed detection in a sugar beet field using discriminant analysis with wavelet transform. These pixel discriminant models showed the possibility of plant classification and weed detection using hyperspectral imaging.

The primary goal of this study was image segmentation between crop and weed for weed detection. First, pixel discriminant models between crop and weed were developed, and model accuracy was validated and discussed. Then, segmented images between crop and weed were generated by applying the pixel discriminant models to each pixel in the hyperspectral images.

\section{MATERIALS AND METHODS}

\section{Hyperspectral imaging and sensing system}

Hyperspectral images were acquired using a hyperspectral camera (ImSpector V10; Specim Ltd., Oulu, Finland) with a spectral wavelength range of 360 to $1010 \mathrm{~nm}$ and a spectral resolution of $10 \mathrm{~nm}$. The camera integrates a spectrograph and a matrix detector, and functions as a line sensor (Fig. 1). Figure 2 shows the output of the hyperspectral camera. The horizontal axis shows the spatial information, and the vertical axis shows the spectral information. Therefore, the direction of the camera must be moved in order to scan a target area.

A portable hyperspectral image capturing system developed by Okamoto et al. (2006a and 2006b) and Ye et al. (2006) was employed in this study. Hyperspectral images were acquired by rotary-motion scanning (vertical direction) of the electrically driven pan head. Acquired images were recorded on digital video tape $(720 \times 480$ pixels $)$ using a digital video recorder (GR-D650; Victor Co., Tokyo, Japan) as video data files.

\section{Image acquisition}

This study was conducted in a soybean field at the Hokkaido University Experimental Farm, Sapporo, Hokkaido, Japan. The target crop was soybean (Glycine max (L.) Merr.), and the target weeds were goosefoot pigweed (Chenopodium album L. var. centrorubrum Makino), small crabgrass (Digitaria violascens Link), field horsetail (Equisetum arvense L.) and pearlwort (Sagina japonica (Sw.) Ohwi). Most of crop were third trifoliate leaf stage, and them length were about $30 \mathrm{~cm}$. Hyperspectral images of the crop and weeds were acquired on September 22, 23 and 24, 
IMAGE SEGMENTATION FOR WEEDING

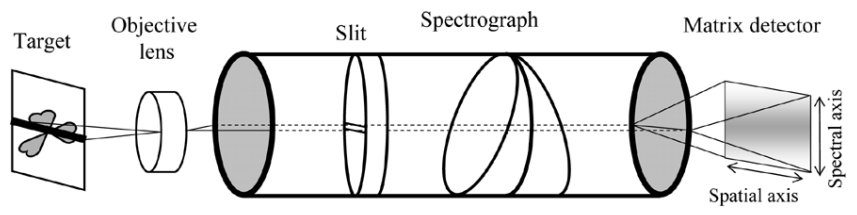

Fig. 1 Mechanism of hyperspectral camera.

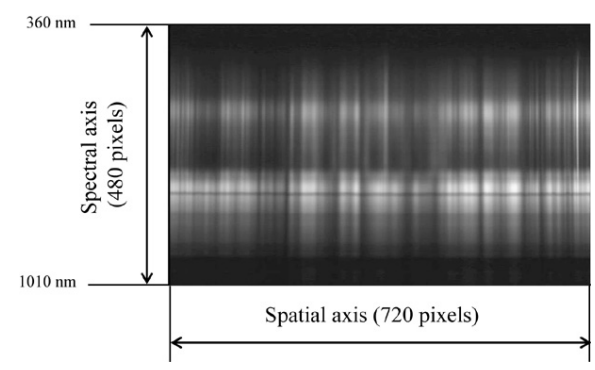

Fig. 2 Output of hyperspectral camera.

2007. Weather conditions were sunny or cloudy.

\section{Model for pixel discrimination}

Hyperspectral images are composed of a large number of pixels, and each pixel contains a spectrum of 60 waveband values. In order to execute image segmentation pixel by pixel, a pixel discriminant model was developed using $\mathrm{R}$ software ( $\mathrm{R}$ Development Core Team, 2007), which is open-source free software for statistical computing.

Sampling of pixel spectra for modeling and validation

The technique of Okamoto et al. (2006a and 2006b) was applied to sampling of pixel spectra from hyperspectral images. Table 1 shows the number of pixel spectra sampled for modeling and validation.

\section{Discrimination between plant and soil}

Prior to discrimination between crop and weed, plants must be extracted from background soil in a hyperspectral image. The difference between plant and soil was observed in the spectrum in the red light range and NIR (near-infrared) light range (Fig. 3). In this study, the waveband values at band No. $30(663 \mathrm{~nm})$ and No. $38(760 \mathrm{~nm})$ were used to discriminate between plant and soil because they were significantly different. Therefore, NDVI (normalized difference vegetation index) calculated from these wavebands was applied to the discrimination. NDVI was calculated by Eq. (1):

$$
N D V I=\frac{N I R-R e d}{N I R+R e d}
$$

Table 1 Number of pixel spectra sampled for modeling and validation.

\begin{tabular}{lccc}
\hline \multicolumn{1}{c}{ Actual species } & Number of samples & Number of pixel samples & Total \\
\hline Soil & 150 (images) & 30 (pixel/image) & 4500 \\
Soybean & 30 (plants) & 30 (pixel/plant) & 900 \\
Goosefoot pigweed & 30 (plants) & 30 (pixel $/$ plant) & 900 \\
Small crabgrass & 30 (plants) & 30 (pixel/plant) & 900 \\
Field horsetail & 30 (plants) & 30 (pixel $/$ plant) & 900 \\
Pearlwort & 30 (plants) & 30 (pixel/plant) & 900 \\
\hline
\end{tabular}




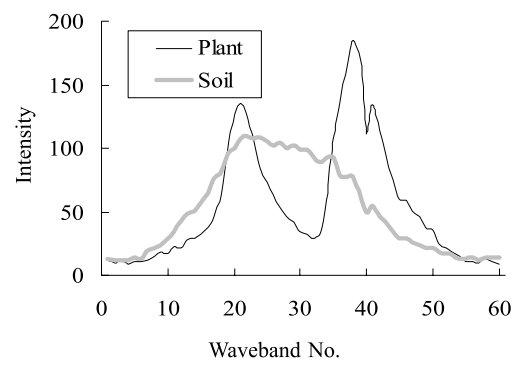

Fig. 3 Pixel spectra of plant and soil. Plant pixel spectrum: Mean pixel spectrum of crop and weeds, Soil pixel spectrum: Mean pixel spectrum of soil.

where,

$N I R=$ value at waveband No. 38

Red $=$ value at waveband No. 30

First, the pixel spectra of all samples were divided in half as modeling and validation datasets. A threshold for discrimination between plant and soil was determined manually by NDVI calculated from the modeling dataset.

Discrimination between crop and weed

A model for pixel discrimination between crop and weed was developed for each weed species. The modeling process was composed of normalization, generation of explanatory variables and discrimination.

Normalization of pixel spectrum

A pixel spectrum was normalized because the total intensity level of each spectrum varied according to the illumination and iris of the lens. A spectrum was transformed by Eq. (2) (Murata et al., 2004; Okamoto et al., 2004 and 2006a), which normalized the original spectrum as the minimum in waveband values was zero, and the mean of waveband values was 1 (Fig. 4).

$$
N_{i}=\frac{S_{i}-S_{\text {min }}}{S_{\text {mean }}-S_{\text {min }}}
$$

where,

$i=$ waveband number

$N_{i}=$ normalized waveband value $(i=1-60)$

$S_{i}=$ original waveband value $(i=1-60)$

$S_{\min }=$ minimum in waveband values

$S_{\text {mean }}=$ mean of waveband values
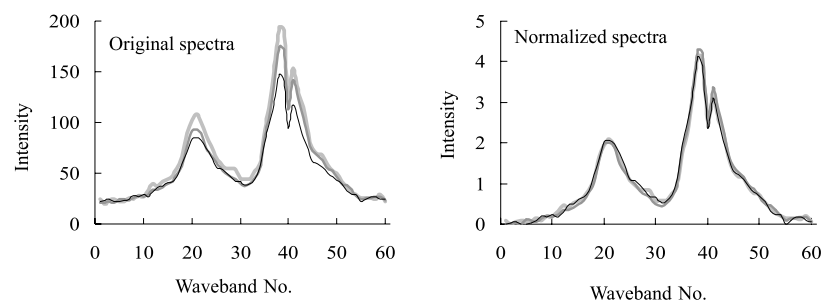

Fig. 4 Normalization of pixel spectrum. 


\section{IMAGE SEGMENTATION FOR WEEDING}

\section{Generation of explanatory variables for discrimination}

Prior to discrimination, the explanatory variables were generated from wavebands. In this study, two different methods (RAW and PCA) were employed for generating variables. In the RAW method, the explanatory variables were simply selected from all wavebands by stepwise selection using Wilks' lambda. This is an index to test for statistical significance, and it ranges from 0 to 1. Stepwise selection was performed using the "greedy. wilks" function (Mardia et al., 1979) in the "klaR" package for R software. In the PCA method, first, waveband values were transformed into principal components by principal component analysis using the "prcomp" function (Mardia et al., 1979; Becker et al., 1988; Venables and Ripley, 2002) in the "stats" package for R software. Next, the explanatory variables were selected from the principal components by stepwise selection in the same manner as the RAW method. The explanatory variables selected by PCA were orthogonal because the principal component analysis performs an orthogonal transformation to a new coordinate system.

Discrimination with explanatory variables

Unlike discrimination between plant and soil, it is difficult to discriminate between crop and weed by simple thresholding because the spectral characteristics of crop and weed are similar. Therefore, linear discriminant analysis (LDA) and a multi-layered neural network (NN) were employed for discrimination between crop and weed. LDA is a statistical method for discrimination between categories that finds a linear discriminant function for multi-category pattern classification. Linear discriminant function is calculated by ratio of variance between categories and variance within the category. It was performed using the "lda" function (Ripley, 1996; Venables and Ripley, 2002) in the "MASS" package for R software. NN is a nonlinear model for various parallel processing of biological neurons in the human brain. Neurons of $\mathrm{NN}$ are contained at each layer with each neurons connected to other neurons in the other layer. In this study, a three layers (input, hidden and output) NN model was developed using a back propagation algorithm. The explanatory variables are found in the input layer. The back propagation algorithm is used as a supervised learning technique of $\mathrm{NN}$. In this technique, the strength of synapse connecting the neurons is modified because the error in the output layer is propagated starting from output layer back to input layer. NN was performed using the "nnet" function (Ripley, 1996; Venables and Ripley, 2002) in the "nnet" package for R software.

Development and validation of discriminant models

Four types of discriminant models (RAW-LDA, RAW-NN, PCA-LDA and PCA-NN) for each weed species were developed and evaluated by cross validation of each model. First, pixel spectra of crop and a target weed species were collected for discrimination between crop and each weed species. Next, they were partitioned into two datasets for modeling and validation. The validation dataset consisted of 30 pixel spectra on a single plant sample chosen from among all plant samples, and the modeling dataset for developing a pixel discriminant model consisted of pixel spectra on the remaining plant samples. Then, the model developed using the modeling dataset was evaluated using the validation dataset. This process was repeated $N$ ( $N$ is the number of plant samples) times with different combinations of datasets. Finally, success rate of validation was calculated based on the number of correct predictions over the total number of tests during the evaluation of the model.

Image segmentation between crop and weed

In this study, the software for image segmentation between crop and weed was developed using Microsoft Visual C\#. This software was based on the object-oriented software framework designed for agricultural hyperspectral image analysis (Okamoto et al., 2006a) and can be used to easily change a pixel discriminant model because of its flexibility for multipurpose use. Figure 5 shows the flow of image segmentation. Segmented images were generated from hyperspectral images by applying the pixel discriminant models. In the segmentation, a hyperspectral image was processed pixel by pixel, and pixel spectra in the image were classified into crop, weed or soil 


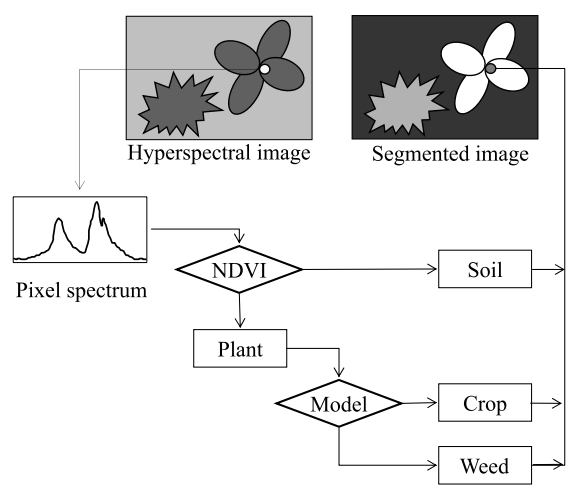

Fig. 5 Flow of image segmentation.

using the pixel discriminant models. Then, the pixels were mapped with different colors depending on the categories.

\section{RESULTS AND DISCUSSION}

\section{Model for pixel discrimination}

Discrimination between plant and soil

Figure 6 shows the NDVI of plant and soil samples in the modeling dataset. NDVI of soil samples ranged from -0.23 to 0.18 , and NDVI of plant samples ranged from 0.30 to 0.77 . Therefore, the threshold for discriminating between soil and plant was determined to be 0.25 . Table 2 shows the validation results of discrimination between soil and plant. Success rates in the validation were $99.9 \%$. Discrimination between soil and plant could be performed with a high degree of accuracy.

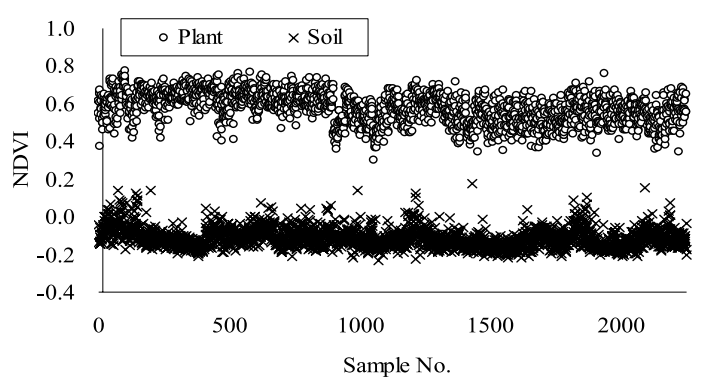

Fig. 6 NDVI of plant and soil samples in modeling dataset.

Table 2 Validation results of discrimination between soil and plant.

\begin{tabular}{llrr}
\hline & \multirow{2}{*}{ Actual category } & \multicolumn{2}{c}{ Estimated category (\%) } \\
\cline { 3 - 4 } & & Soil & Plant \\
\hline Soil & & 99.9 & 0.1 \\
Plant & ( Soybean ) & 0.0 & 100.0 \\
& (Goosefoot pigweed) & 0.0 & 100.0 \\
& (Small crabgrass) & 0.0 & 100.0 \\
& (Field horsetail) & 0.0 & 100.0 \\
& (Pearlwort) & 0.0 & 100.0 \\
\hline
\end{tabular}




\section{IMAGE SEGMENTATION FOR WEEDING}

\section{Discrimination between crop and weed}

Figure 7 shows the top 15 wavebands selected for the models using RAW. Most of the selected wavebands belonged to the green or NIR light range. Therefore, it was considered that these ranges were the most important for discrimination between crop and weed. Figure 8 shows the relationship between the number of explanatory variables and the success rate of RAW-LDA and RAW-NN. The success rates gradually increased, and then became almost constant. In this study, the number of explanatory variables for discriminant models was determined manually from the change in success rate. The number of explanatory variables was determined as 4 to 10 , and the number of explanatory variables of RAW-NN was more than that of RAW-LDA. In the validation results, while the success rates of the models between soybean and goosefoot pigweed were under $90 \%(86.7-89.3 \%)$, the success rates of the other models were more than $90 \%(92.9-96.8 \%)$ (Table 3). The accuracy of RAW-NN models was higher than that of RAW-LDA.

Figure 9 shows the relationship between the number of explanatory variables and the success rate of PCA-LDA and PCA-NN. The success rates of these models changed as in the models using RAW. The number of explanatory variables was determined as 4 to 7 , and the number of explanatory variables of many PCA-NN was more than that of PCA-LDA. In the validation results, while the success rate of the PCA-LDA model between soybean and goosefoot pigweed was $89.3 \%$, the success rates of the other models were more than $90 \%$ (90.2-99.1\%) (Table 3). The accuracy of
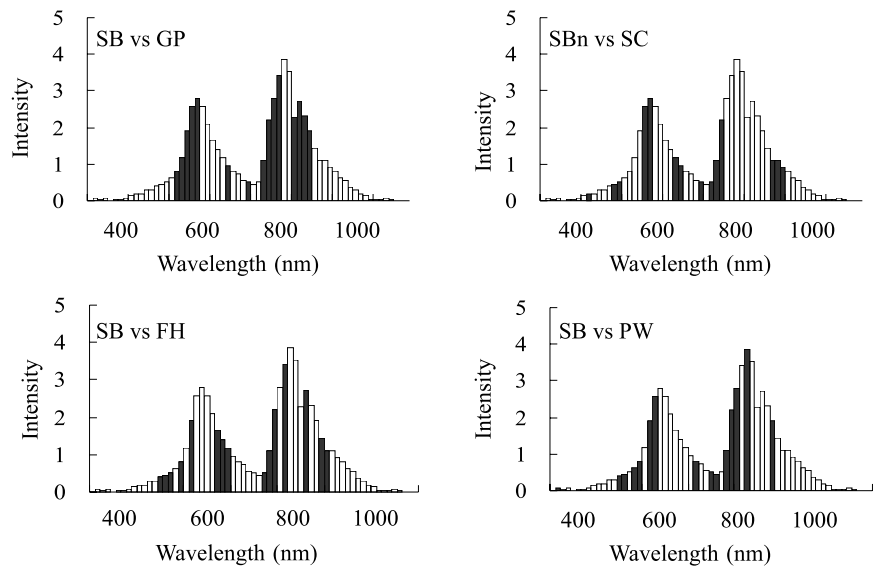

Fig. 7 Top 15 wavebands selected for models using RAW. SB: Soybean, GP: Goosefoot pigweed, SC: Small cragrass, FH: Field horsetail, PW: Pearlwort.
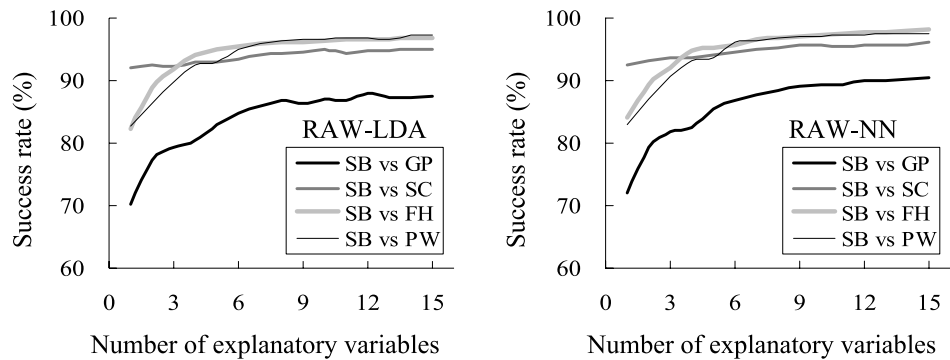

Fig. 8 Relationship between number of explanatory variables and success rate in models using RAW. SB: Soybean, GP: Goosefoot pigweed, SC: Small cragrass, FH: Field horsetail, PW: Pearlwort. 


\section{Y. SUZUKI ET AL.}

Table 3 Validation results of discrimination between crop and weed.

\begin{tabular}{|c|c|c|c|c|c|c|c|c|}
\hline \multirow[b]{2}{*}{ Target } & \multicolumn{2}{|c|}{ RAW-LDA } & \multicolumn{2}{|c|}{ RAW-NN } & \multicolumn{2}{|c|}{ PCA-LDA } & \multicolumn{2}{|c|}{ PCA-NN } \\
\hline & $\mathrm{N}$ & $\begin{array}{l}\text { Success } \\
\text { rate }(\%)\end{array}$ & $\mathrm{N}$ & $\begin{array}{c}\text { Success } \\
\text { rate }(\%)\end{array}$ & $\mathrm{N}$ & $\begin{array}{c}\text { Success } \\
\text { rate }(\%)\end{array}$ & $\mathrm{N}$ & $\begin{array}{l}\text { Success } \\
\text { rate }(\%)\end{array}$ \\
\hline SB vs GP & 8 & 86.7 & 10 & 89.3 & 6 & 89.3 & 6 & 90.2 \\
\hline $\mathrm{SB}$ vs $\mathrm{SC}$ & 4 & 92.9 & 7 & 95.1 & 5 & 93.3 & 6 & 95.3 \\
\hline $\mathrm{SB}$ vs FH & 6 & 95.5 & 8 & 96.8 & 4 & 94.9 & 7 & 99.1 \\
\hline SB vs PW & 7 & 95.9 & 6 & 96.1 & 5 & 97.0 & 4 & 97.6 \\
\hline
\end{tabular}

SB: Soybean, GP: Goosefoot pigweed, SC: Small cragrass, FH: Field horsetail, PW: Pearlwort, N: Number of explanatory variables.
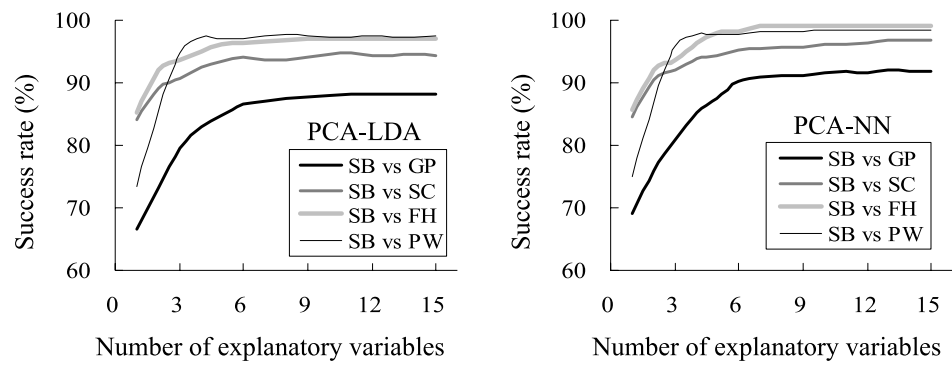

Fig. 9 Relationship between number of explanatory variables and success rate in models using PCA. SB: Soybean, GP: Goosefoot pigweed, SC: Small cragrass, FH: Field horsetail, PW: Pearlwort.

the PCA-NN models was higher than that of the PCA-LDA model.

Except for the RAW-LDA and PCA-LDA models between soybean and small crabgrass, the number of explanatory variables of models using PCA was fewer than that of the models using RAW. The accuracy of models using PCA was higher than that of RAW; the reason for this was considered to be because the explanatory variables of PCA were orthogonal principal components condensing the information of waveband values. However, an estimation model for hyperspectral imaging should be developed considering both accuracy and processing speed because a hyperspectral image contains a large number of pixel spectra. Explanatory variables for models using PCA were selected from principal components calculated from all wavebands. The processing speed of models using PCA was slower than that of models using RAW, which simply selected some wavebands from among all the wavebands. Therefore, in terms of processing speed, the models using RAW are superior to those using PCA.

\section{Image segmentation between crop and weed}

Figure 10 shows examples of image segmentation between soybean and each weed species applying PCA-LDA models. In these images, pixels identified as crop (soybean) are shown in white, weed in gray and soil in black. The majority of pixels were identified correctly. Some pixels, especially at the edges of leaves, were misidentified, and it may be difficult to discriminate completely between crop and weed. However misidentified pixels could be removed using spatial processing such as noise reduction and majority rule, therefore, this technique will be applied to weed detection. Although most of the hyperspectral images were segmented correctly, there were some cases of incorrect segmentation. Figure 11 shows examples of poor image segmentation applying PCA-LDA models for discrimination between soybean and goosefoot pigweed to the hyperspectral image. In this case, many pixels in the shadowed areas of soybean leaves were misidentified. In this study, most pixel spectrum samples were collected from the sunny areas on plant leaves and employed for the development and validation of discriminant models. Therefore, these models did not consider identification of pixel spectra on shady leaves. To resolve this, pixel 

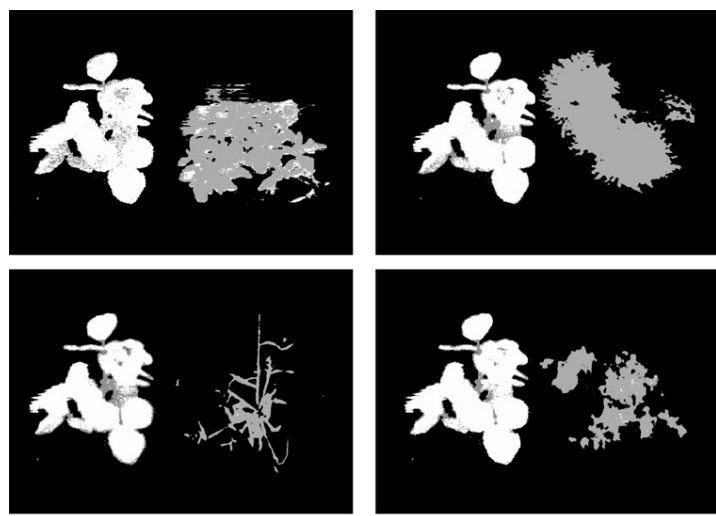

(crop: white, weed: gray, soil: black)

Fig. 10 Examples of image segmentation. (crop: white, weed: gray, soil: black)

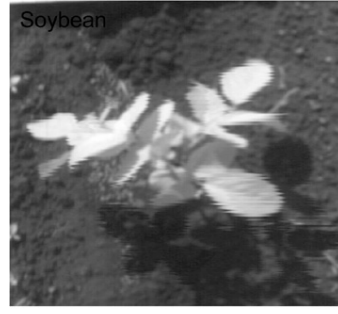

NIR band image ( $748 \mathrm{~nm})$

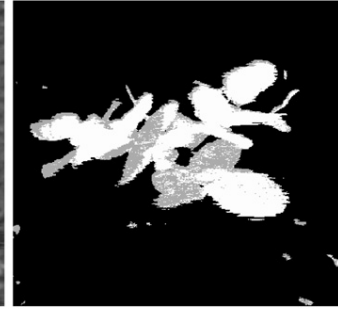

Segmented image (crop: white, weed: gray, soil: black)

Fig. 11 Examples of poor image segmentation.

spectra in both sunny and shady areas must be collected for development and validation of the models.

In this study, the target was not intertwined with other plant species. Therefore, few pixels which have misidentified could be corrected using simple spatial processing. In the case where the plant is intertwined with others, higher spatial processing technique such as labeling would be needed to discriminate between them.

\section{CONCLUSION}

Physical weed control is safer than chemical methods because it solves such problems as pollution of food production and natural environment, and damage to farmers' health. However, most of the work involved in this method is mainly performed manually at present. Consequently, there is an increasing demand for a robotic weeding system to achieve efficient weed control. The primary goal of this study was image segmentation between crop and weed for weed detection. First, pixel discriminant models between crop and weed were developed and validated. Then, segmented images were generated by applying the pixel discriminant models to each pixel in the hyperspectral images.

Hyperspectral images are composed of a large number of pixel spectra. Image segmentation was executed pixel by pixel. First, each pixel spectrum was extracted from the image. Next, the pixel spectrum was classified into soil and plant. If the pixel spectrum was identified as plant, it 


\section{Y. SUZUKI ET AL.}

was classified into crop and weed. In the pixel discriminant model between soil and plant, simple NDVI thresholding was employed. The pixel discriminant model between crop and weed consisted of normalization, generation of explanatory variables and discrimination. A plant spectrum was normalized to compensate the total intensity level of each spectrum. The generation of explanatory variables for discrimination was tested using two different methods (RAW or PCA). Discrimination was also tested using two different methods (LDA or NN). In this study, four types of discriminant models (RAW-LDA, RAW-NN, PCA-LDA and PCA-NN) were developed and validated. Finally, segmented images were generated from hyperspectral images using software based on the object-oriented software framework that has flexibility for multipurpose use.

In the validation results of pixel discrimination between soil and plant, discrimination was performed with a high degree of accuracy, $99.9 \%$. In the validation results of pixel discrimination between crop and weed, the success rates of the models between soybean and goosefoot pigweed were lower $(86.7-90.2 \%)$ than the other models, but the success rates of other models were more than $90 \%(92.9-99.1 \%)$. As for accuracy, NN models were superior to LDA, and models using PCA were superior to those using RAW. However, in terms of processing speed, models using RAW were superior to those using PCA. For further study, modeling requires consideration of both accuracy and processing speed. In the results of image segmentation between crop and weed, the majority of hyperspectral images were segmented correctly. Some pixels, especially at the edges of leaves, were misidentified, but they could be removed using spatial processing. However, in some cases of discrimination between soybean and goosefoot pigweed, many pixels in the shadowed areas of soybean leaves were misidentified.

In conclusion, this study demonstrated the possibility of weed detection using hyperspectral imaging.

This study was funded by a grant from the Ministry of Education, Culture, Sports, Science and Technology, Japan (No. 17208022). We would also like to thank the staff of the Experimental Farm, Field Science Center for Northern Biosphere, Hokkaido University.

\section{REFERENCES}

Bajwa, S. G., Tian, L. F. 2001. Aerial CIR remote sensing for weed density mapping in a soybean field. Transactions of the ASAE 44: 1965-1974.

Becker, R. A., Chambers, J. M., Wilks, A. R. 1988. The New S Language. Wadsworth \& Brooks/Cole, California.

Goel, P. K., Prasher, S. O., Landry, J. A., Patel, R. M., Viau, A. A. 2003. Hyperspectral image classification to detect weed infestations and nitrogen status in corn. Transactions of the ASAE 46: 539-550.

Karimi, Y., Prasher, S. O., Bonnell, R. B., Dutilleul, P., Goel, P. K. 2005. Classification accuracy of discriminant analysis, artificial neural networks, and decision trees for weed and nitrogen stress detection in corn. Transactions of the ASAE 48: 1261-1268.

Iino,Y., Okamoto, H., Kataoka, T., Hata, S. 2005. Distinction crops from weeds by using hyper spectral imaging. (in Japanese with English abstract) Journal of Hokkaido Branch of the Japanese Society of Agricultural Machinery 45: 21-26.

Mardia, K. V., Kent, J. T., Bibby, J. M. 1979. Multivariate Analysis, Academic Press, London.

Murata, T., Okamoto, H., Kataoka, T., Hata, S. 2004. Crop growth sensing using hyperspectral imaging. (in Japanese with English abstract) Journal of Hokkaido Branch of the Japanese Society of Agricultural Machinery 44: 45-49.

Okamoto, H., Murata, T., Kataoka, T., Hata, S. 2004. Weed detection using hyperspectral imaging. Proceeding of the 7-8 October Conference, Automation Technology for Off-road Equipment: 47-55.

Okamoto, H., Sakai, K., Murata, T., Kataoka, T., Hata, S. 2006a. Object-oriented software framework developed for agricultural hyperspectral imaging analysis. (in Japanese with English abstract) Agricultural Information Research 15: 103-112. 


\section{IMAGE SEGMENTATION FOR WEEDING}

Okamoto, H., Sakai, K., Murata, T., Kataoka, T., Hata, S. 2006b. Development of remote sensing software based on hyperspectral imaging framework. (in Japanese with English abstract) Agricultural Information Research 15: 219-230.

Okamoto, H., Murata, T., Kataoka, T., Hata, S. 2007. Plant classification for weed detection using hyperspectral imaging with wavelet analysis. Weed Biology and Management 7: 31-37.

R Development Core Team. 2007. R: A language and environment for statistical computing. R Foundation for Statistical Computing, Vienna. Available from URL: http://www.R-project.org/ (Cited 28 July 2007).

Ripley, B. D. 1996. Pattern recognition and neural networks. Cambridge University Press, Cambridge.

Steward, B. L., Tian, L. F. 1999. Machine-vision weed density estimation for real-time, outdoor lighting conditions. Transactions of the ASAE 42: 1897-1909.

Tang, L., Tian, L. F., Steward, B. L. 2003. Classification of broadleaf and grass weed using gabor wavelets and an artificial neural network. Transactions of the ASAE 46: 1247-1254.

Terawaki, M., Kataoka, T., Okamoto, H., Hata, S. 2002. Distinction between sugar beet and weeds for development of automatic thinner and weeding machine of sugar beet. Proceeding of the 26-27 July Conference, Automation Technology for Off-road Equipment: 129-136.

Venables, W. N., Ripley, B. D. 2002. Modern applied statistics with S, Ed. 4. Springer, New York.

Yang, C. C., Prasher, S. O., Landry, J., Ramaswamy, H. S. 2002. Development of neural networks for weed recognition in corn fields. Transactions of the ASAE 45: 859-864.

Yang, C. C., Prasher, S. O., Goel, P. K. 2004. Differentiation of crop and weeds by decision-tree analysis of multi-spectral data. Transactions of the ASAE 47: 873-879.

Ye, X., Sakai, K., Gorciano, L. O. 2006. A ground-base hyperspectral imaging system for characterization vegetation spectral features. Preprints of 3rd IFAC International Workshop on Bio-robotics: 165-170. 